\title{
KKMC-hh: A Precision Event Generator for EW Radiative Corrections in Hadron Scattering
}

\section{S.A. Yost ${ }^{* \dagger}$}

Author The Citadel, The Military College of South Carolina

E-mail: scott.yost@citadel.edu

\section{S. Jadach}

Institute of Nuclear Physics IFJ-PAN, Cracow, Poland

E-mail: stanislaw.jadachecern.ch

\section{B.F.L. Ward}

Baylor University

E-mail: bfl_wardebaylor.edu

\section{Z. Wạs}

Institute of Nuclear Physics IFJ-PAN, Cracow, Poland

Email: wasmecern.ch

\begin{abstract}
KKMC-hh is a precision event-generator for $Z$ production and decay in hadronic collisions, which applies amplitude-level exponentiation to both initial and final state photon radiation, including perturbative residuals through order $\alpha^{2} L$, together with electroweak matrix element corrections. We present results showing the effect of multi-photon radiation for cuts motivated by a recent ATLAS $W$-mass analysis. We also show preliminary untuned comparisons of the electroweak corrections of KKMC-hh to those of HORACE, which includes order $\alpha$ corrections with exponentiated final-state photon radiation.
\end{abstract}

13th International Symposium on Radiative Corrections 24-29 September, 2017

St. Gilgen, Austria

\footnotetext{
*Speaker.

${ }^{\dagger}$ This presentation was supported by a grant from the Citadel Foundation.
} 


\section{Introduction}

A precise calculation of vector boson production is essential for interpreting electroweak precision data from the LHC and anticipated future hadron colliders. For example, a recent analysis [1] of the $7 \mathrm{TeV}$ data from ATLAS yielded a measure of the $W$ boson mass of

$$
M_{W}=80370 \pm 7 \text { (stat.) } \pm 11 \text { (exp.syst.) } \pm 14 \text { (mod.syst.) } \mathrm{MeV}=80370 \pm 19 \mathrm{MeV} .
$$

The modeling systematic error of $14 \mathrm{MeV}$ is the largest contribution to the uncertainty. With increasing statistics, it will become important to reduce this modeling error. Certain aspects of the $W$ production and decay systematics were estimated by comparing to analogous systematics for $Z / \gamma^{*}$ production and decay, so improving the electroweak precision for the $Z$ calculation improves the $W$ mass result as well.

ATLAS has reported [2] a measurement of the $Z$ differential spectra with a 2.5 per mille statistical error in the peak region, and comparable NLO EW corrections at central rapidities when FSR is unfolded using PHOTOS. [3, 4, 5] Per-mille level statistical errors in differential spectra for $Z / \gamma^{*}$ production and decay are also reported by ATLAS in Ref. [7], again unfolding FSR effects using PHOTOS. The analysis of Ref. [8] shows a $0.2 \%$ statistical error in the differential spectra for $Z / \gamma^{*}$ production for $7 \mathrm{TeV}$ data at the LHC. Unfolding the FSR with PHOTOS (cross-checked with SHERPA [6]) results in a $0.3 \%$ error in the $P_{\mathrm{T}}$ spectrum for muon pairs, and a $0.1 \%$ error for electron pairs. CMS has also reported per mille level statistical errors in the $Z / \gamma^{*} P_{\mathrm{T}}$ spectrum, with a per mille level estimate of the systematic error due to FSR. [9]

These increasingly precise analyses raise the question of what per-mille level higher-order EW corrections should be included in a careful analysis of the systematics. With KKMC-hh, $[10,11]$ it is possible to answer this question by unfolding $\mathscr{O}\left(\alpha^{2} L\right) \mathrm{EW}$ corrections from the data. This would give a more complete test of the contributions relevant to precision EW measurements. We will present results from KKMC-hh showing the result of calculating multi-photon effects at various levels of precision for cuts inspired by the ATLAS $W$-mass analysis, as well as comparisons to other programs, including HORACE $[12,13]$, which calculates NLO EW corrections with exponentiated FSR.

\section{The Physics of KKMC-hh}

KKMC-hh [10, 11] is based on the LEP-era event generator KKMC [15] for $e^{+} e^{-} \rightarrow f \bar{f}+n \gamma$, where $f \bar{f}$ represents a final state fermion pair, for CMS energies from $2 m_{\tau}$ to $1 \mathrm{TeV}$. The precision tag for LEP2 was $0.2 \%$. The MC structure is based on CEEX, [14, 15, 16, 17] an amplitude-level analog of YFS exponentiation, [18] and includes residuals through order $\alpha^{2} L$ where $L-\ln \left(s / m_{e}^{2}\right)$ is a relevant "big logarithm." Electroweak matrix element corrections are included via DIZET 6.21, from the semi-analytical program ZFITTER [25]. DIZET calculates vacuum polarization factors for the photon and $Z$ propagators, and adds form-factor corrections to the vector coupling and an angle-dependent form-factor to incorporate the effect of box diagrams. The correction factors are tabulated at the beginning of a run. Tau decay is implemented using TAUOLA. [19, 20, 21, 22]

Version 4.22 [17] of KKMC supports quark initial states, and a modified version of this is incorporated into KKMC-hh with the addition of support for selecting the quarks via PDFs, using 
an LHAPDF[23] interface. KKMC-hh uses the adaptive MC program FOAM [24] to to generate the quark momentum fractions $x_{i}$, the total ISR energy, and the quark flavor using a crude distribution which is constructed during an initialization phase.

CEEX was introduced [16] to overcome limitations of traditional YFS exponentiation, which suffered from a proliferation of interference terms, limiting its ability to reach the desired $0.2 \%$ precision tag for LEP2. CEEX works at the level of spinor helicity amplitudes, which greatly facilitates calculating effects such as ISR-FSR interference (IFI). The IFI effects in KKMC-hh can be switched on or off, allowing an assessment of their importance. Also, it is possible to switch to traditional YFS exponentiation, called EEX, for comparison purposes. The level of residuals can also be selected, so that the effect of higher-order corrections in $\alpha$ can be assessed.

KKMC-hh can export its events in an LHE [26] event record to be showered externally, or the events can be showered internally using HERWIG 6.5. [27] In the following sections, we will present both showered and unshowered results. There are also plans to implement a mode in which KKMC-hh can reweight events from an any external QCD shower generator, and add photons. The combination of NLO QCD with EW corrections will take advantage of the fact that to a good approximation, these corrections factorize. $[28,29]$ The direct inclusion of NLO QCD into KKMChh is also anticipated, using the KrkNLO [30] scheme.

\section{KKMC-hh Results for the ATLAS Acceptance}

As noted in the introduction, the recent ATLAS measurement of $M_{W}$ estimated some aspects of the $W$ production and decay systematics, such as the uncertainty in the momentum resolution scale, using the analogous systematics for $Z / \gamma^{*}$ events. The uncertainty in the EW corrections in $Z$ production thus contributes to the $W$ mass measurement's systematics. In this section, we illustrate the size of the higher order EW effects now available via KKMC-hh using the cuts applied in the ATLAS analysis. More details of this analysis may be found in Ref. [31] The ATLAS cuts [1] on the invariant mass and transverse momentum of the dilepton pair are

$$
80 \mathrm{GeV}<M_{\ell \ell}<100 \mathrm{GeV}, P_{\mathrm{T}}^{\ell \ell}<30 \mathrm{GeV},
$$

while the transverse momentum and pseudorapidity of each lepton is constrained by

$$
P_{T}^{\ell}>25 \mathrm{GeV},\left|\eta_{\ell}\right|<2.5 \text {. }
$$

In these tests, we generate $10^{8}$-event samples for $7 \mathrm{TeV}$ collisions using MSTW2008 PDFs [32] and shower with HERWIG6.5. We compare the best $\mathscr{O}\left(\alpha^{2} L\right)$ CEEX implementation (labeled CEEX2) to several more limited models: $\mathscr{O}\left(\alpha^{2} L\right)$ CEEX without IFI (initial-final interference), $\mathscr{O}(\alpha)$ EEX (labeled EEX1), and $\mathscr{O}(\alpha)$ EEX without ISR (initial-state radiation). Table 1 table shows the cross-sections with and without the cuts. All of the calculations are compatible to a fraction of a per mille, The cut cross section with ISR off shows a per mille difference relative to the full CEEX2 result, while all other differences are a fraction of a per mille. 


\begin{tabular}{|l|c|c|c|c|}
\hline & uncut $(\mathrm{pb})$ & Difference & cut $(\mathrm{pb})$ & Difference \\
\hline CEEX2 & 844.74 & $\times$ & 280.36 & $\times$ \\
CEEX2 (no IFI) & 844.97 & $+0.03 \%$ & 280.31 & $-0.02 \%$ \\
EEX1 & 844.45 & $-0.03 \%$ & 280.38 & $+0.007 \%$ \\
EEX1 (no ISR) & 844.97 & $+0.03 \%$ & 280.64 & $+0.10 \%$ \\
\hline
\end{tabular}

Table 1. Total Cross Sections With and Without ATLAS Cuts. Differences are shown relative to CEEX2.

Figures 1 and 2 compare differential spectra for the transverse momentum and pseudorapidity of the $\mu^{-}$in $Z / \gamma^{*}$ production with decay to muon pairs for the ATLAS cuts. While the contribution of ISR to the total cross section was at the per mille level, the effect on distributions is much greater, especially in the $P_{\mathrm{T}}$ distribution where a several percent ISR effect is seen. Initial-final interference (IFI) is a fractional per mille effect.
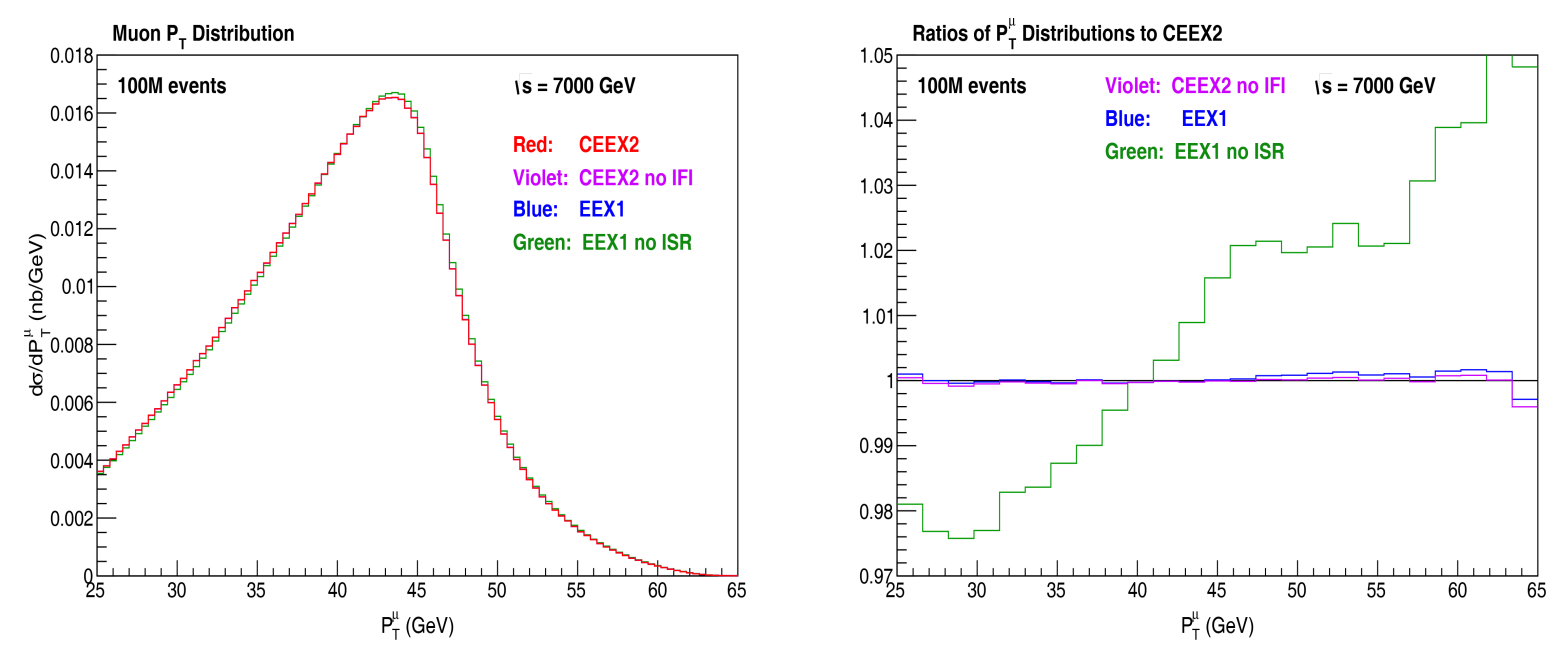

Figure 1: Muon Transverse Momentum Distributions and Ratios

Figures 3 - 5 compare the dimuon invariant mass, transverse momentum, and rapidity for these cuts. Photon distributions are shown in figures 6 and 7. Percent-level ISR effects are also seen in the dimuon invariant mass spectrum. Initial-final interference is a fractional per mille effect in all cases. In calculations were per mille level accuracy is required, all of the contributions in KKMC-hh should be taken into account. 

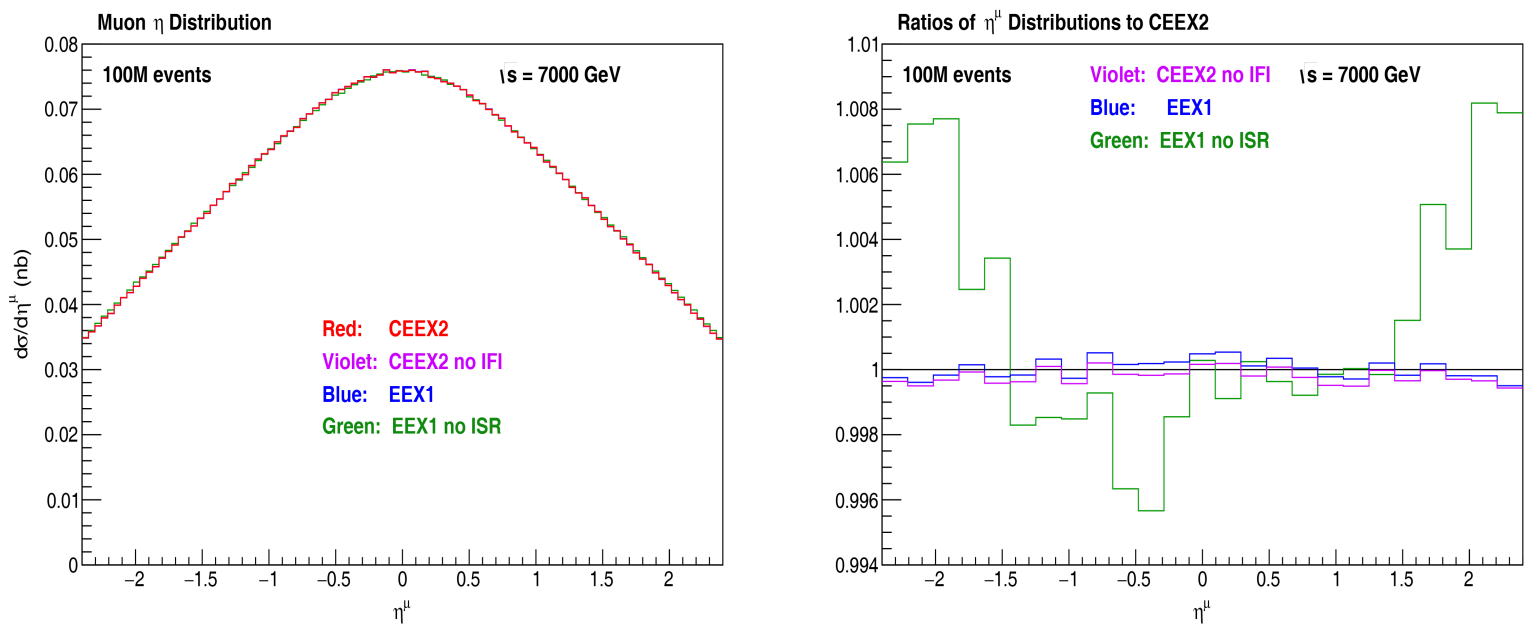

Figure 2: Muon Pseudorapidity Distributions and Ratios
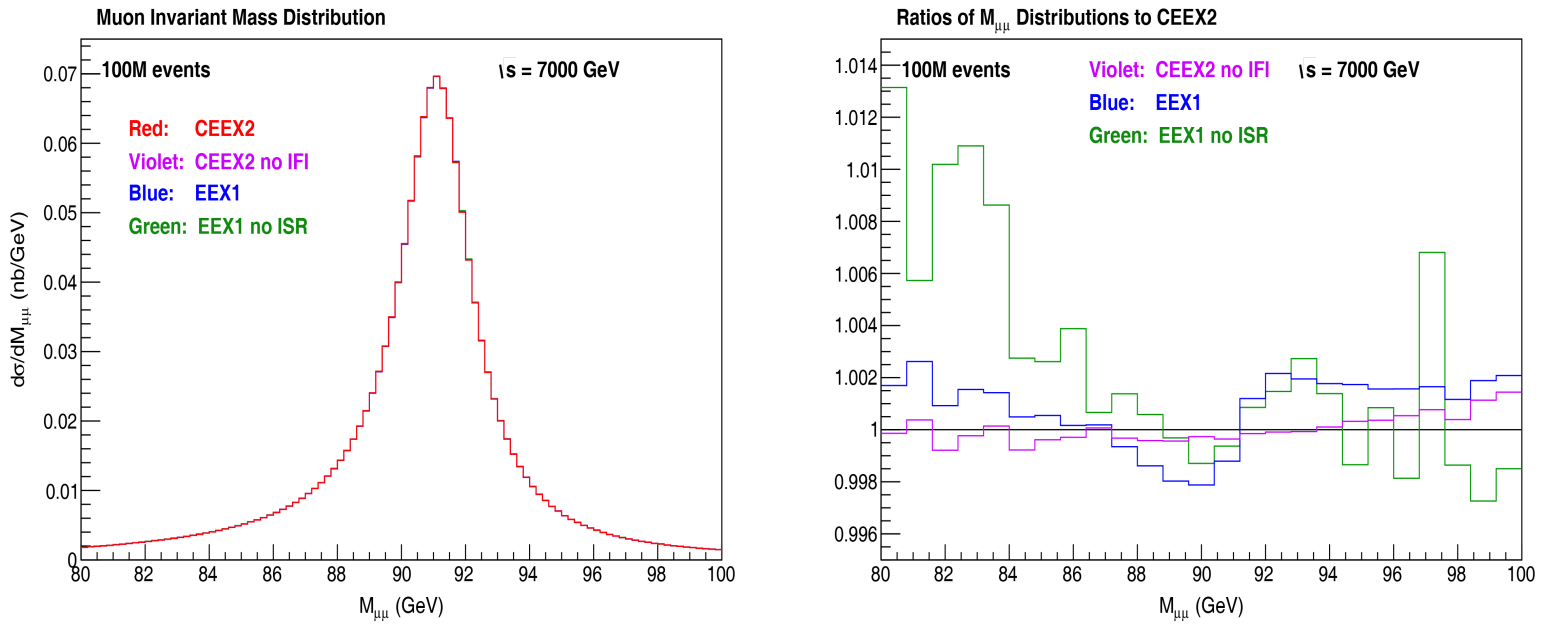

Figure 3: Dimuon Invariant Mass Distributions and Ratios 

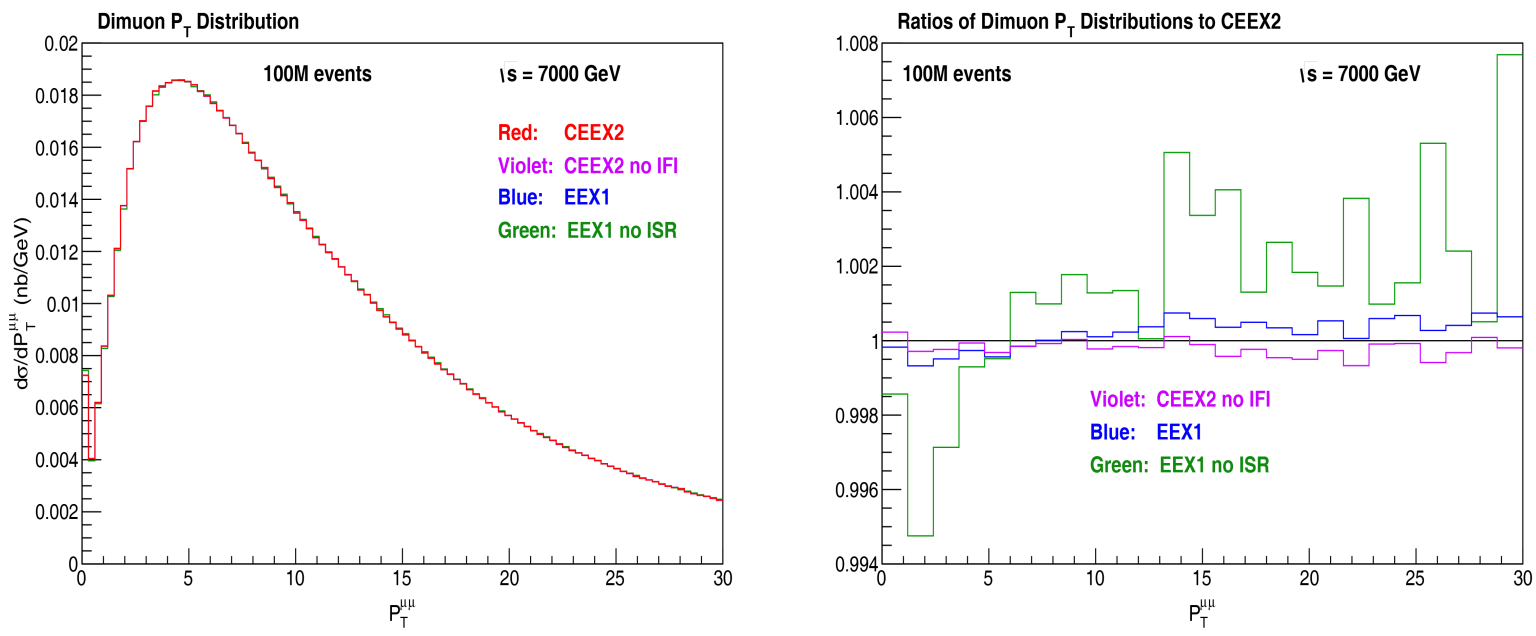

Figure 4: Dimuon Transverse Momentum Distributions and Ratios
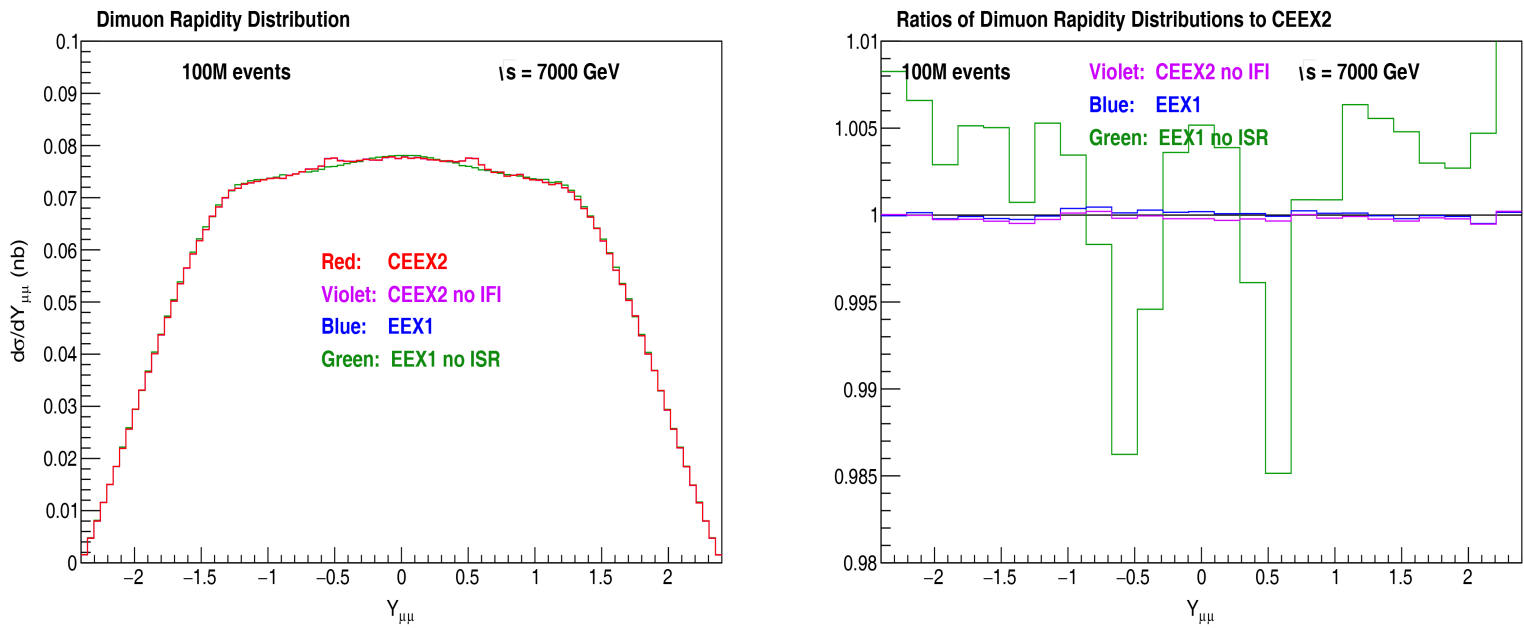

Figure 5: Dimuon Rapidity Distributions and Ratios 

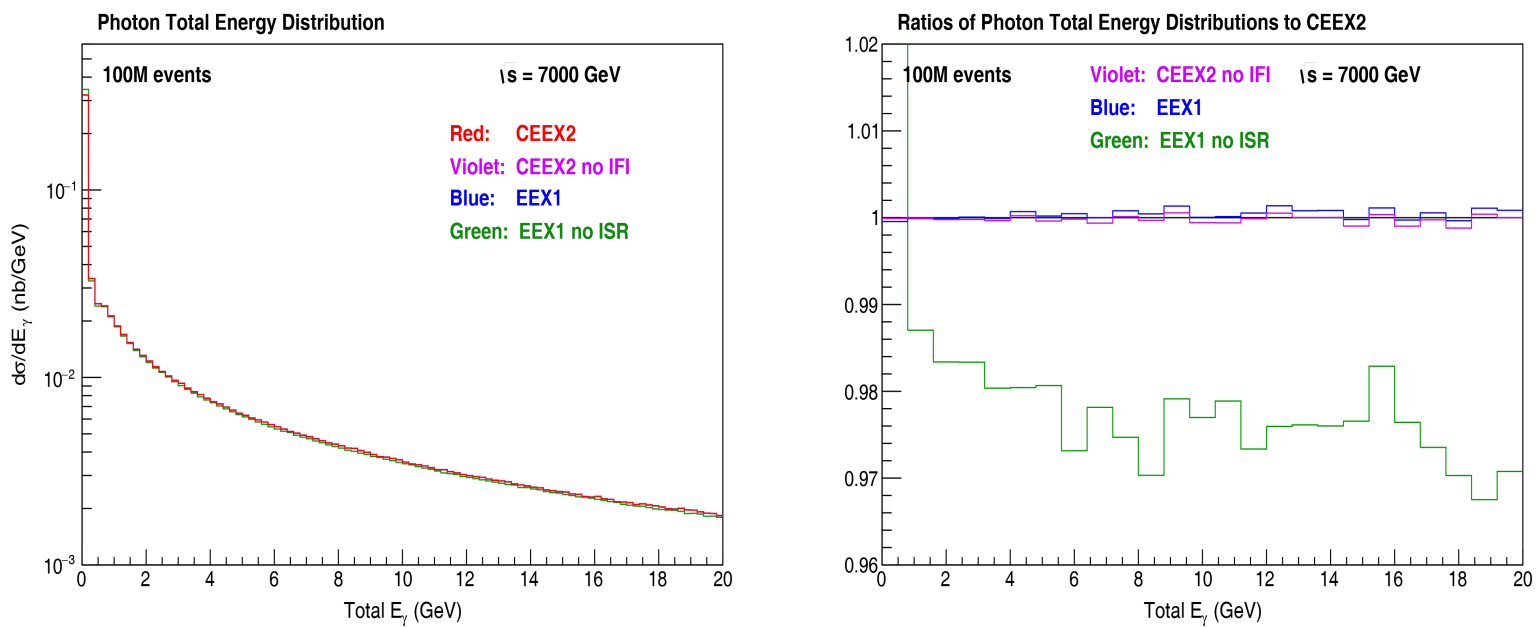

Figure 6: Total Radiated Photon Energy
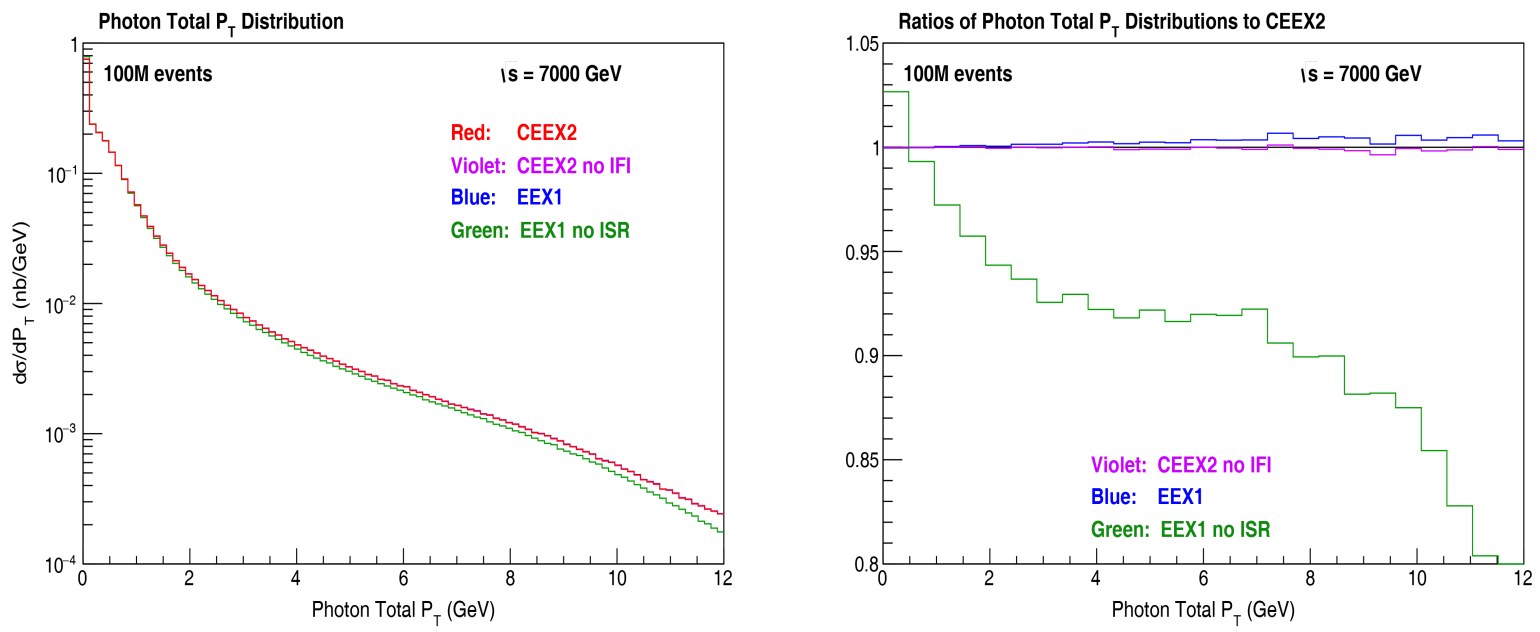

Figure 7: Total Transverse Momentum of Radiated Photons 


\section{Comparisons of KKMC-hh and HORACE}

In this section, we show untuned comparisons of muon production at $8 \mathrm{TeV}$ with MSTW PDFs and a cut $50 \mathrm{GeV}<M_{q \bar{q}}<200 \mathrm{GeV}$ on the generated quark pair and no QCD shower. We compare the KKMC-hh results to HORACE, $[12,13]$ which includes $\mathscr{O}(\alpha)$ EW corrections and exponentiated final state photon emission. The HORACE events are generated in the "best" EW scheme with exponentiated FSR. In this mode, HORACE should agree closely with KKMC-hh in its CEEX $\mathscr{O}(\alpha)$ exponentiated mode with ISR turned off. Unshowered events from HERWIG6.5 are shown for a comparison without EW corrections. The samples for HORACE and HERWIG6.5 have $10^{8}$ events, while the KKMC-hh sample has $25 \times 10^{6}$ events. We include two KKMC-hh results: the full result including ISR, FSR, and IFI at $\mathscr{O}\left(\alpha^{2} L\right)$ and a restriction to FSR only at $\mathscr{O}(\alpha)$ (CEEX1), which is should be similar to HORACE. The HORACE Born-level result, without photons, is also compared. Table 2 shows differences between the total cross sections and the full KKMC-hh result. All comparisons are without a QCD shower.

\begin{tabular}{|l|c|c|c|}
\hline MC & EW Corrections & $\sigma(\mathrm{pb})$ & Difference \\
\hline KKMC-hh & CEEX2 & $993 \pm 1$ & $\times$ \\
KKMC-hh & CEEX1 (no ISR) & $991 \pm 1$ & $-0.20 \%$ \\
HORACE & $\mathscr{O}(\alpha)$ exp. & $1009.6 \pm 0.4$ & $+1.7 \%$ \\
HORACE & Born $($ no $\gamma$ 's) & $1025.2 \pm 0.4$ & $+3.2 \%$ \\
HERWIG6.5 & Born $($ no $\gamma$ 's) & $1039.6 \pm 0.2$ & $+4.7 \%$ \\
\hline
\end{tabular}

Table 2. Total Cross Section Comparisons and Difference Relative to CEEX2

Figure 8 compares the $\mu^{-}$transverse momentum and pseudorapidity distributions. Figure 9 compares the dimuon invariant mass distribution and the multiplicity of photons having at least 1 $\mathrm{GeV}$ of energy. There is a small normalization difference in the distributions, but they appear to be as compatible as can be expected prior to a precise tuning of the parameters.
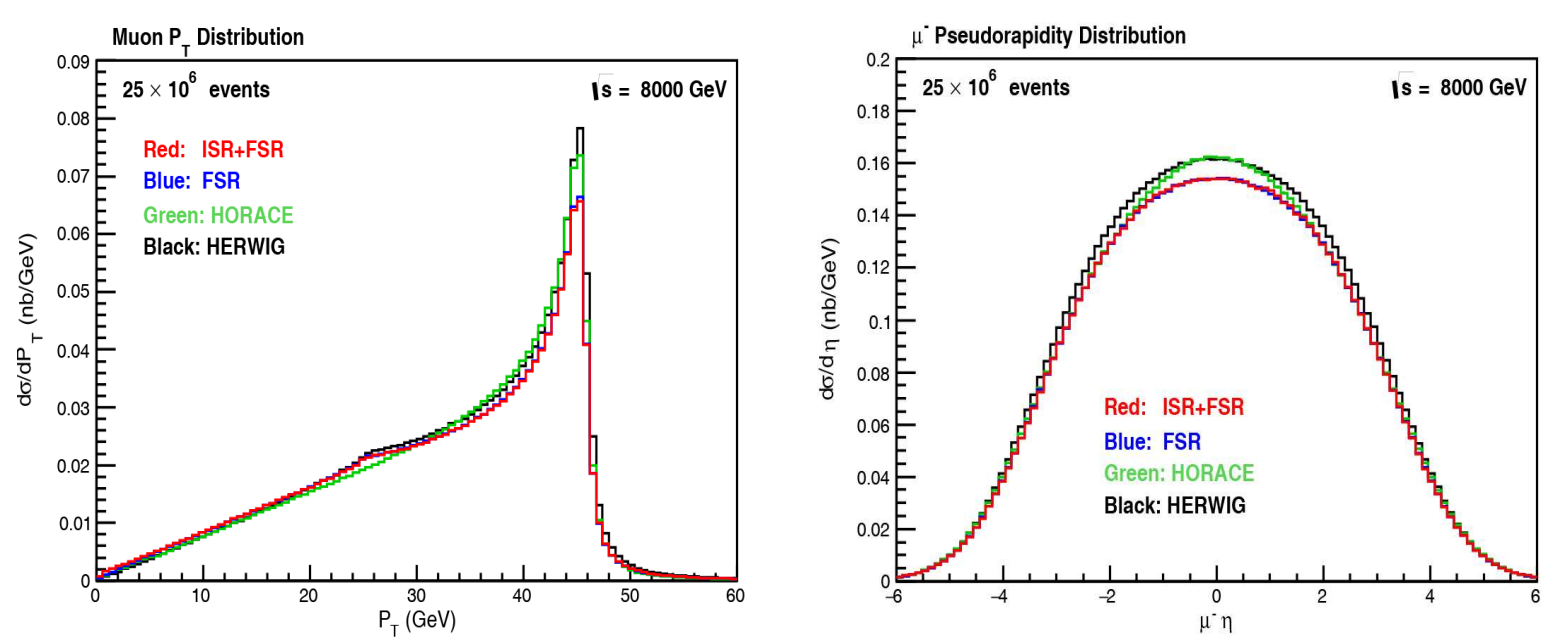

Figure 8: Muon Transverse Momentum and Pseudorapidity Distributions 

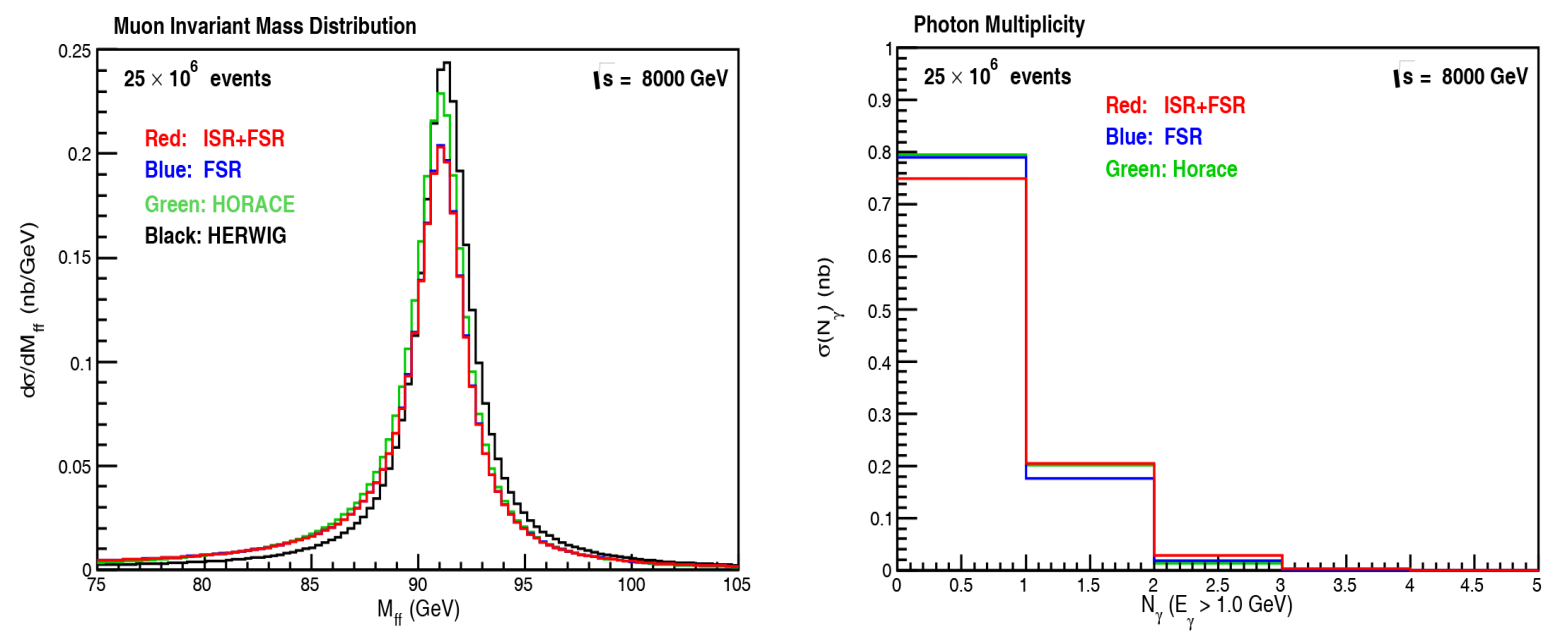

Figure 9: Dimuon Invariant Mass and Photon Multiplicity (for $E_{\gamma}>1 \mathrm{GeV}$ ) Distributions

\section{Conclusions}

The results for ATLAS cuts show that for per mille level studies, ISR, IFI, and exact $\mathscr{O}\left(\alpha^{2} L\right)$ corrections should all be included for a conservative estimate of the precision tag. KKMC-hh is available on request for such studies. Untuned comparisons to HORACE show a promising level of agreement when the programs are compared at a compatible precision level $(\mathscr{O}(\alpha)$ with exponentiated FSR radiation). Tuned comparisons to the results in ref. [33] should be available in the near future.

Further developments are under-way, including improvements to the interface which will allow KKMC-hh to operate either as a primary generator, creating events to be showered subsequently (the present mode), or to operate as an add-on generator, to add photons and appropriate reweighting to events generated by any available parton shower, including NLO showers. It is also anticipated that NLO QCD will be added directly to KKMC-hh using the KrkNLO scheme. [30] Other future enhancements may include adding the effect of additional fermion pairs and updating the EW matrix element corrections using a version of SANC. [34, 35] It should be straightforward to create a version of KKMC-hh for $W$ production, but this is not yet under development.

\section{References}

[1] M. Aaboud et al., CERN-EP-2016-305 (2017) [arXiv:1701.07240].

[2] M. Aaboud et al., CERN-EP-2016-272 (2016) [arXiv:1612.03016].

[3] E. Barberio, B. van Eijk and Z. Wạs, Comput. Phys. Commun. 66 (1991) 115.

[4] E. Barberio and Z. Wa̧s, Comput. Phys. Commun. 79 (1994) 291.

[5] P. Golonka and Z. Wạs, Eur. Phys. J. C45 (2006) 97 [hep-ph/0506026].

[6] T. Gleisberg et al., JHEP 02 (2009) 007 [arXiv:0811.4622].

[7] G. Aad et al., Eur. Phys. J. C76 (2016) 291 [arXiv:1512.02192]. 
[8] G. Aad et al., JHEP 09 (2014) 145 [arXiv:1406.3660].

[9] K. Khachatryan et al., CERN-PH-EP/2015-059, CMS-SMP-13-013 (2015) [arXiv:1504.03511].

[10] S. Jadach, B.F.L. Ward, Z. Wa̧s, and S.A. Yost, Phys. Rev. D94 (2016) 074006 [arXiv:1608.01260].

[11] S. Yost and B.F.L. Ward, PoS (LL2016) (2016) 062 [arXiv:1606.09032].

[12] C.M. Carloni Calame, G. Montagna, O. Nicrosini and M. Treccani, Phys. Rev. D69 (2004) 037301; ibid., JHEP 0505 (2005) 019.

[13] C.M. Carloni Calame et al., JHEP 0612 (2006) 016; ibid., JHEP 0710 (2007) 109.

[14] S. Jadach, B.F.L. Ward, and Z. Wạs, Comput. Phys. Commun. 79 (1994) 503.

[15] S. Jadach, B.F.L. Ward, and Z. Wạs, Comput. Phys. Commun. 130 (2000) 260 [hep-ph/9912214].

[16] S. Jadach, B.F.L. Ward, and Z. Wa̧s, Phys. Rev. D63 (2001) 113009 [hep-ph/0006359].

[17] S. Jadach, B.F.L. Ward, and Z. Wa̧s, Phys. Rev. D88 (2013) 114022 [arXiv:1307.4037].

[18] D.R. Yennie, S.C. Frautschi and H. Suura, Ann. Phys. 13 (1961) 379; K.T. Mahanthappa, Phys. Rev. 126 (1962) 329.

[19] S. Jadach, J.H. Kühn and Z. Wa̧s, Comput. Phys. Commun. 64 (1990) 275.

[20] M. Jeżabek, Z. Wạs, S. Jadach and J.H. Kühn, Comput. Phys. Commun. 70 (1992) 69.

[21] S. Jadach, Z. Wass, R. Decker and J.H. Kühn, Comput. Phys. Commun. 76 (1993) 361.

[22] P. Golonka et al., Comput. Phys. Commun. 174 (2006) 818 [hep-ph/0312240].

[23] A. Buckley, et al., Eur. Phys. J. C75 (2015) 3, 132.

[24] S. Jadach, Comput. Phys. Commun. 152 (2003) 55.

[25] D. Bardin et al., Comput. Phys. Commun. 133 (2001) 229 [hep-ph/9908433].

[26] E. Boos et al., Generic User Process Interface for Event Generators, (2001) [hep-ph/0109068].

[27] G. Corcella et al., JHEP 0101 (2001) 010 [hep-ph/0011363].

[28] A. Denner, S. Dittmaier, T. Kasprzik and A. Mück, Eur. Phys. J. C73 (2013) 2297 [arXiv:1211.5078].

[29] S. Dittmaier, A. Huss, and C. Schwinn, Nucl. Phys. B855 (2014) 318 [arXiv:1403.3216]; ibid., Nucl. Phys. B904 (2016) 216 [arXiv:1511.08016].

[30] S. Jadach et al., Phys. Rev. D87 034029 [arXiv:1103.5015]; ibid., Acta Phys. Polon. B46 (2015) 2089; ibid., JHEP 10 (2015) 052 [arXiv:1503.06849].

[31] S. Jadach, B.F.L. Ward, Z. W as, and S.A. Yost, Systematic Studies of Exact $\mathscr{O}\left(\alpha^{2} L\right) C E E X E W$ Corrections in a Hadronic MC for Precision Z/ $\gamma^{*}$ Physics at LHC Energies (2017), arXiv:1707.06502.

[32] A.D. Martin, W.J. Stirling, R.S. Thorne and G. Watt, Eur. Phys. J. C63 (2009) 189 [arXiv:0901.0002].

[33] S. Alioli et al., Eur. Phys. J. C77 (2017) 280 [arXiv:1606.02330].

[34] A. Andonov et al., Comput. Phys. Commun. 174 (2006) 481 [hep-ph/0411186]; Erratum: ibid. 177 (2007) 623 .

[35] A. Andonov et al., Comput. Phys. Commun. 181 (2010) 181 [arXiv:0812.4207]. 